MEI, II, Vol. 4, nº 6, pág. 43

\title{
Digital.csic: el impacto de la ciencia en abierto
}

MEI

II, vol. 4

$n^{\circ} 6$
M. Dolores Martínez Santiago

Consejo Superior de Investigaciones Científicas
Recibido el $10-07-2013$

Aceptado en 25-09-2013

\section{Resumen}

Desde la Oficina Técnica de la Unidad de Recursos de Información Científica para la Investigación (URICI) se están llevando a cabo mejoras en el repositorio institucional Digital.CSIC con el objeto de analizar la visibilidad y el impacto de los trabajos depositados a través de estadísticas, citas y otros indicadores. Para optimizar el tiempo de depósito, han desarrollado la pasarela ConCiencia que permite trasvasar los datos bibliográficos y el texto completo al repositorio de forma rápida, tanto es así que a partir del mes de su inauguración ha habido un gran incremento de depósitos en el repositorio.

\section{Palabras clave}

Digital.CSIC, Acceso abierto, Pasarela ConCiencia, Visibilidad, Impacto, Estadísticas, CSIC.

Digital.csic: the impact of science on open

\section{Abstract}

From the technical office of the Unit of Resource of Scientific Information for Research (URICI) improvements in the institutional repository, Digital.CSIC are being conducted in order to analyze the visibility and impact of the works deposited through statistics, citations and other indicators. To optimize the time of deposit, they have developed a gateway (ConCiencia), that allows you to download the bibliographic data and the full text to the repository in a fast way, so much so that starting from the month of its inauguration there has been an big increase of deposits in the repository.

\section{Keywords}

Digital.CSIC, Open access, Gateway ConCiencia, Visibility, Impact, Statistics, CSIC.

\section{1.-Objetivo}

El objetivo de este artículo es realizar un análisis cuantitativo para obtener un balance del depósito de la producción científica en el repositorio institucional Digital.CSIC, así como dar a conocer las novedades desarrolladas por la Oficina Técnica como la pasarela ConCiencia, aplicación utilizada para trasvasar los registros al repositorio, la incorporación de información bibliométrica a través de APIs (Application Programming Interface), las mejoras realizadas en el repositorio para obtener más visibilidad y, por último, las nuevas líneas de trabajo para este año.

\section{2.-El repositorio del CSI}

El Consejo Superior de Investigaciones Científicas (CSIC) es la mayor institución pública dedicada a la investigación en España y la tercera de 
Europa. Está adscrito al Ministerio de Economía y Competitividad, a través de la Secretaría de Estado de Investigación, Desarrollo e Innovación, y su objetivo principal es desarrollar y promover investigaciones en beneficio del progreso científico y tecnológico.

El CSIC firmó el 31 de enero de 2006 la Declaración de Berlín (2003) con el compromiso de ofrecer en acceso abierto los resultados de las investigaciones realizadas en sus centros e institutos. Este compromiso se ha materializado a través de dos vías:

- Por una parte, la vía dorada en la que ha estado trabajando durante estos últimos tres años la Unidad de Recursos de Información Científica para la Investigación (URICI) encargada de la dirección y gestión de la Red de Bibliotecas del CSIC, la cual ha firmado varios acuerdos con importantes editoriales como Biomed Central, Chemistry Central, Springer Open y la Royal Society of Chemistry con el fin de subvencionar el 57'5\% de las cuotas de publicación en acceso abierto de sus autores, a cambio de que éstos depositen su producción científica de los últimos tres años en el repositorio institucional siempre que las políticas editoriales lo permitan. Por la situación económica que vivimos en estos momentos, estos fondos son escasos y limitados, por lo que solo se financia un artículo por año y por autor.

Durante el año pasado, se beneficiaron 55 investigadores del CSIC publicando mayoritariamente en revistas de Biomed Central, de los cuales el $70 \%$ cumplió con el compromiso exigido.

- La segunda vía ha sido la creación en 2008 del repositorio institucional Digital.CSIC (vía verde) cuyo objetivo es organizar, archivar, preservar a largo plazo y difundir en acceso abierto la producción científica de los investigadores del CSIC.

El archivo de documentos en un repositorio de acceso abierto aporta importantes beneficios, ya que permite devolver a la sociedad la ciencia que financia, consigue una mayor y rápida difusión de los resultados de la investigación con lo cual genera más impacto, incrementa la visibilidad no sólo de la institución sino también la del investigador, cuanto mayor es el número de lectores mayor son las citaciones con lo que facilita futuras financiaciones, permite preservar los contenidos a largo plazo, y proporciona el acceso gratuito a la investigación de un país, entre otras ventajas.

Ambas estrategias se complementan con el objetivo de facilitar y mejorar el acceso y la difusión de la información científica.

Una de las principales fuentes de financiación de los proyectos de investigación del CSIC es la Comisión Europea. El año pasado hubo 65 proyectos sujetos al 7 Programa Marco y 17 proyectos afectados por el mandato del Consejo Europeo de 
Investigación. Este mandato requiere por parte de los equipos investigadores que difundan la versión pdf editorial o el post-print de su trabajo resultante (artículos, monografías o datos crudos) en repositorios de acceso abierto en un plazo de seis meses después de la publicación y doce meses si se trata del área de Humanidades y Ciencias sociales. Un tercio de estos proyectos cumplieron con dicha difusión.

\section{3.-Ámbito y evolución del Digital.CSIC}

Digital.CSIC es un repositorio multidisciplinar que cubre todos los campos del conocimiento organizado en nueve comunidades que recogen toda clase de tipología documental.

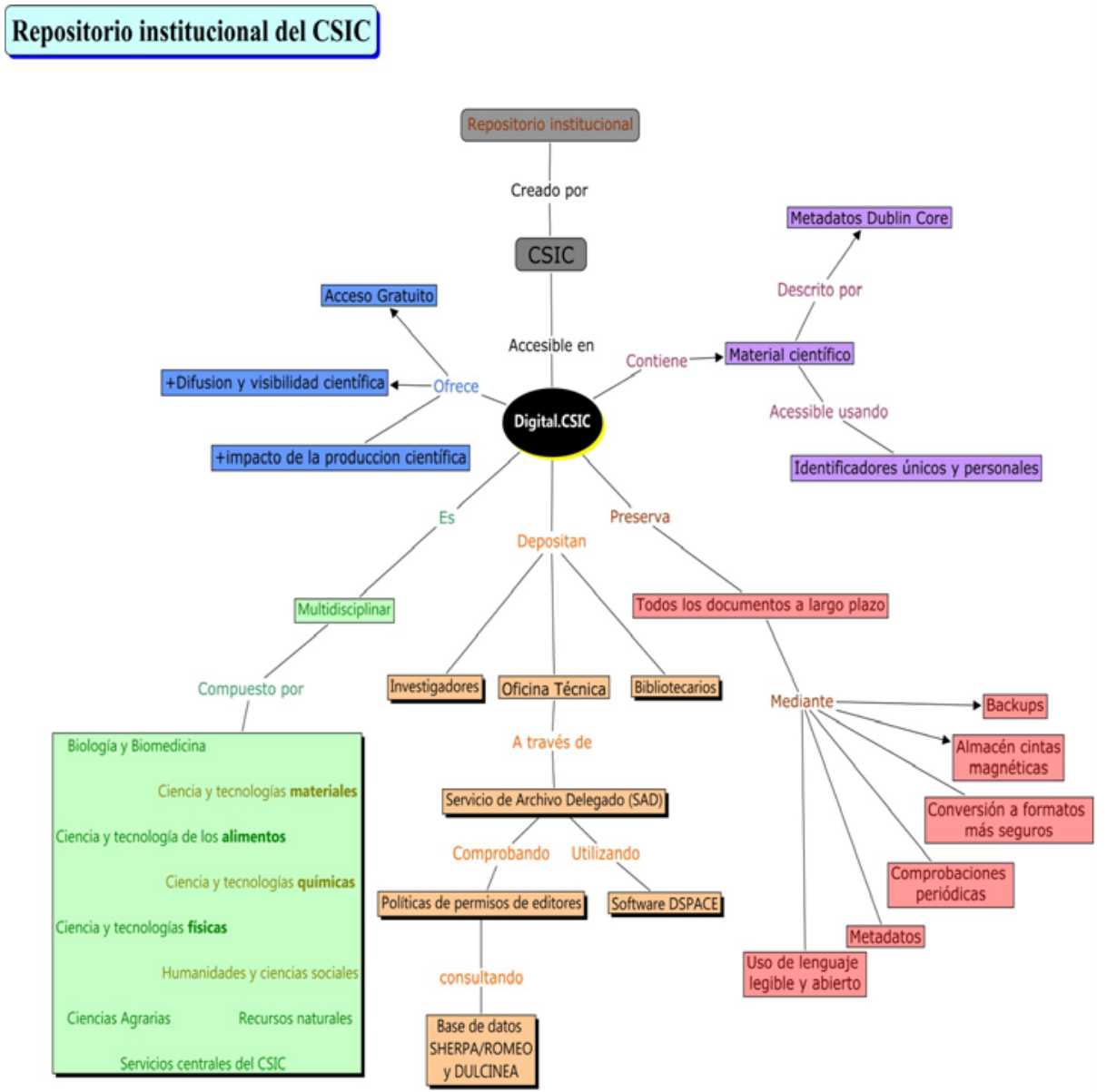

Fig. 1. Mapa conceptual sobre el repositorio Digital.CSIC

(Fuente: M.D. Martínez y A. Fuster)

El depósito de documentos en el repositorio se puede realizar mediante el auto-archivo, en el que los propios investigadores archivan sus trabajos o a través del Servicio de Archivo Delegado (SAD) efectuado por las bibliotecas y la Oficina Técnica de la URICI, donde se realiza el depósito de centros e institutos adheridos al Plan $100 \%$ Digital, este proyecto permite ofrecer diversos servicios a centros/institutos que no tienen personal bibliotecario. El $97 \%$ de los trabajos depositados en 
Digital.CSIC durante el año 2012 se realizó a través del SAD.

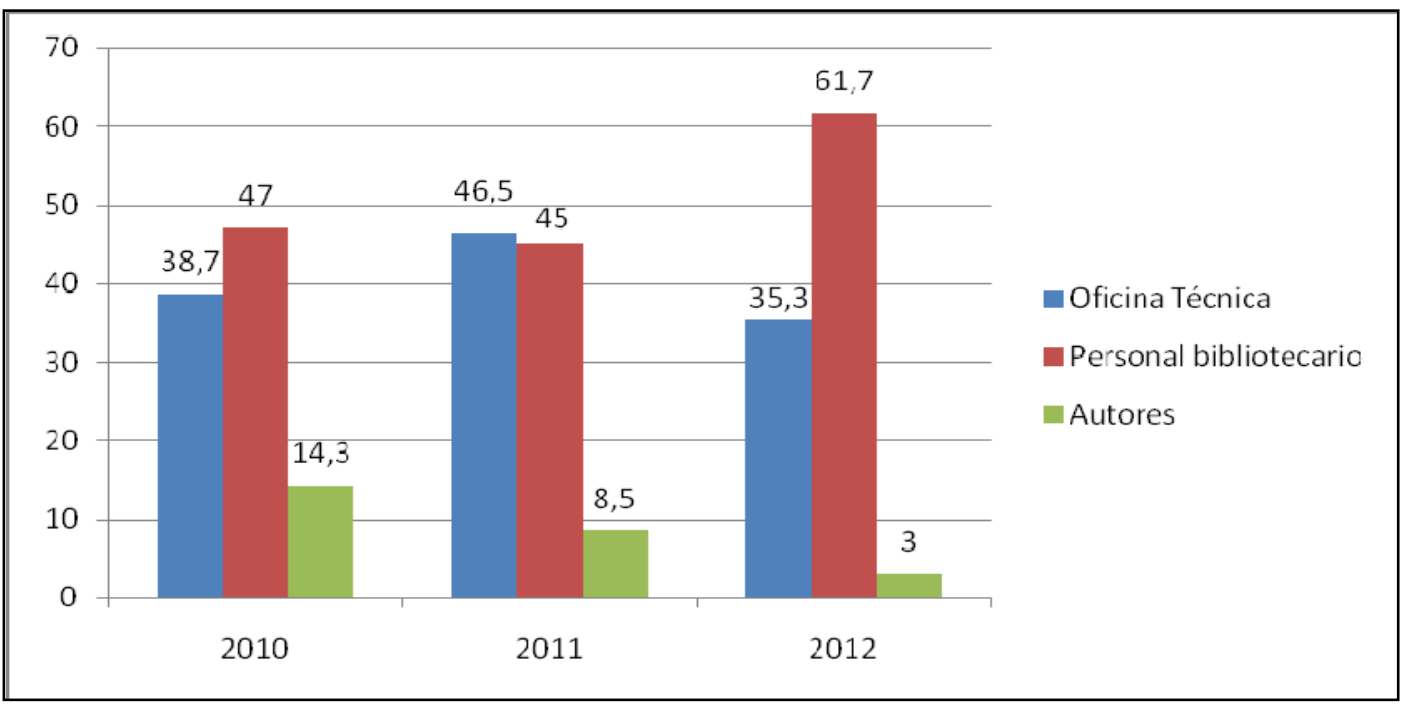

Fig. 2. Depósito en Digital.CSIC durante el periodo 2010-2012

El trabajo que se realiza en la Oficina Técnica se centra en cargar los artículos publicados en revistas científicas durante los tres últimos años, depositar los trabajos afectados por diversos mandatos de acceso abierto, y resolver las dudas de los investigadores y del personal bibliotecario.

En Digital.CSIC se han depositado más de diecinueve mil registros, de los que un $58 \%$ están a texto completo, con lo que se ha incrementado respecto al año anterior en más de siete mil registros $(38 \%)$. Con lo que al cierre del año pasado se hacía un recuento de un total de 59.837 trabajos depositados

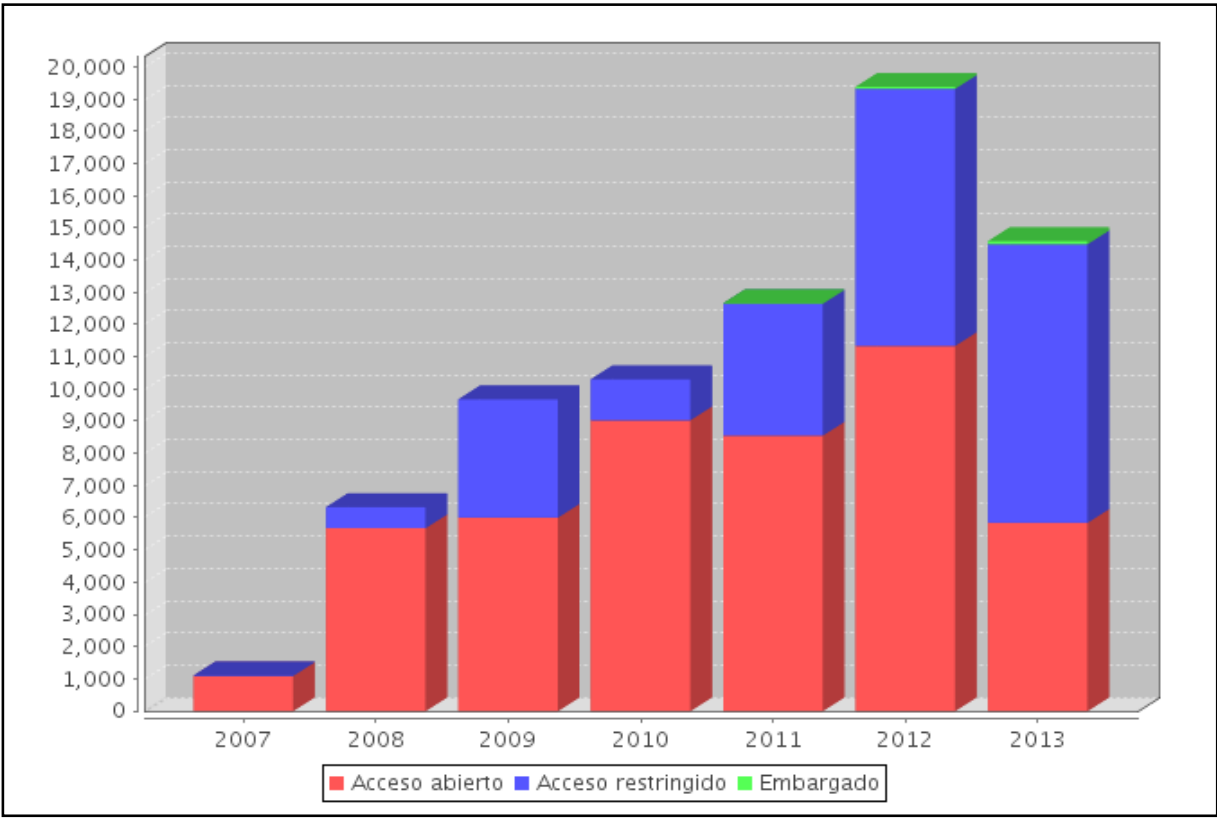

Fig. 3. $\mathrm{N}^{\mathrm{o}}$ de registros depositados en Digital.CSIC por año 
Las visualizaciones realizadas en las páginas de Digital.CSIC han alcanzado más de tres millones y medio en el año 2012 y más de cinco millones en descargas de ficheros, en total un 62\% más de descargas con respecto al año 2011. Como media anual, el $25 \%$ de las descargas se debe a la actividad automática de los motores de búsqueda, agregadores y otros indizadores. Los países que más ficheros se descargaron del repositorio fueron Rusia, Canadá y Cuba. En cuanto a la visualización predominan Estados Unidos, España y Latinoamérica. Estas estadísticas demuestran el incremento de visibilidad que ofrece el repositorio y el impacto que pueden alcanzar los trabajos depositados.

Aparte de las estadísticas de descargas y visualización ofrecidas por el repositorio, la producción científica depositada alimenta automáticamente las páginas de las webs de institutos, portales temáticos, proyectos de investigación, y personales de los propios investigadores.

\section{4.-Mejoras recientes en el repositorio}

Desarrollada por la Oficina Técnica e inaugurada en el mes de junio de 2012, la pasarela ConCiencia es una aplicación que permite volcar a Digital.CSIC los datos y los textos completos de la producción científica, docente y divulgativa contenida en ella e incorpora tareas de captura, organización, descripción y análisis de la producción junto con las de su difusión y preservación digital. Esta aplicación sólo es accesible a través de la intranet para los bibliotecarios encargados del SAD y para los administradores del repositorio.

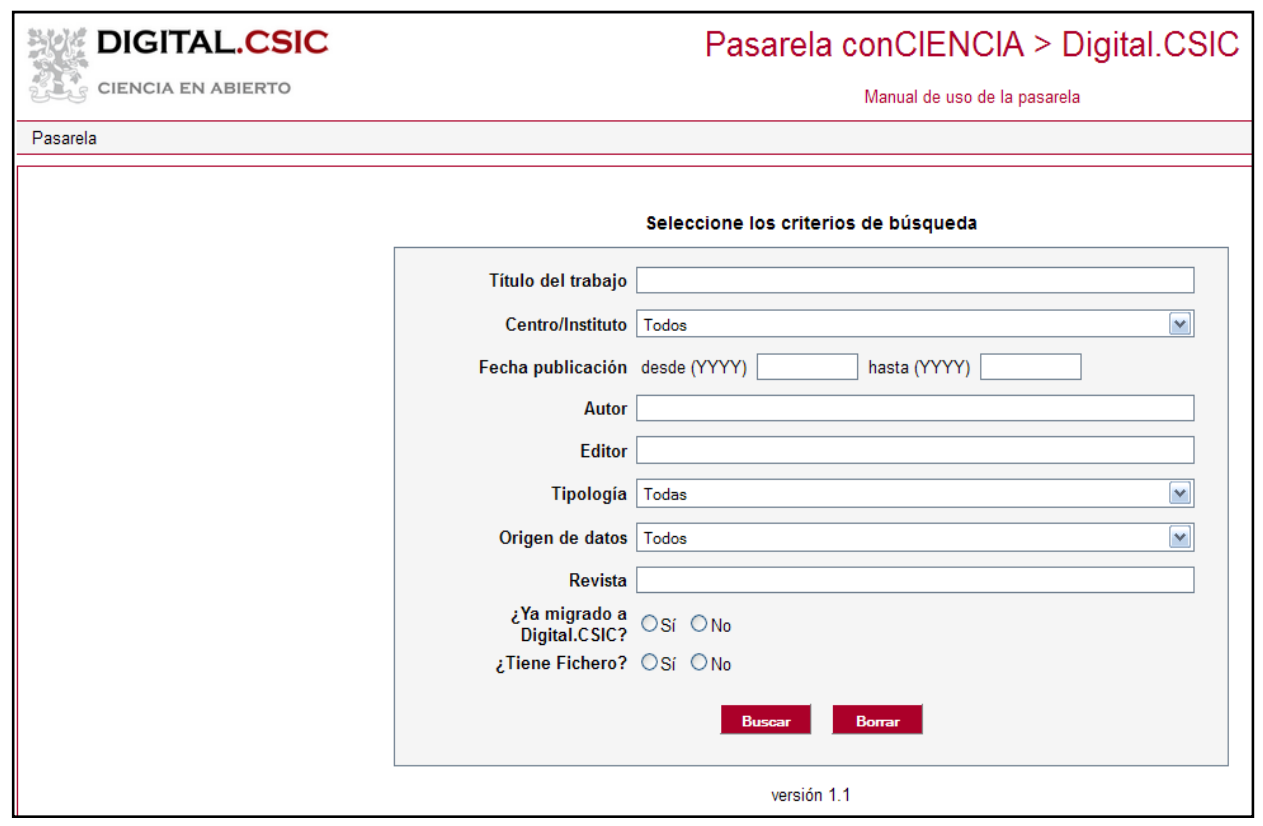

Fig. 4. Pasarela ConCiencia > Digital.CSIC 
Aunque la pasarela necesita algunas mejoras en las que ya se está trabajando, se puede comprobar a través de las estadísticas del repositorio que ha habido un aumento de depósitos desde su inauguración, esto se debe al ahorro de tiempo al hacer el trasvase en comparación con el método tradicional: para empezar existen menos campos que rellenar y algunos datos ya están reseñados en la pasarela como el título, los autores, la tipología documental, los centros/institutos a los que pertenece el trabajo con la opción al final del trasvase de realizar el mapeo, el fichero de la licencia y el de acceso restringido está incluido cuando seleccionas esa opción, posee una ayuda para enlazar con la web de Sherpa/Romeo para conocer la política editorial y un índice para la normalización de los nombres de las editoriales.

En el caso de que no estén en dicho índice se envía un formulario a la Oficina Técnica con el nombre de la editorial para que sea incluido, en el caso de que el fichero no contenga la versión apropiada según la política editorial, hay dos casillas de verificación para aceptar o no ese fichero.

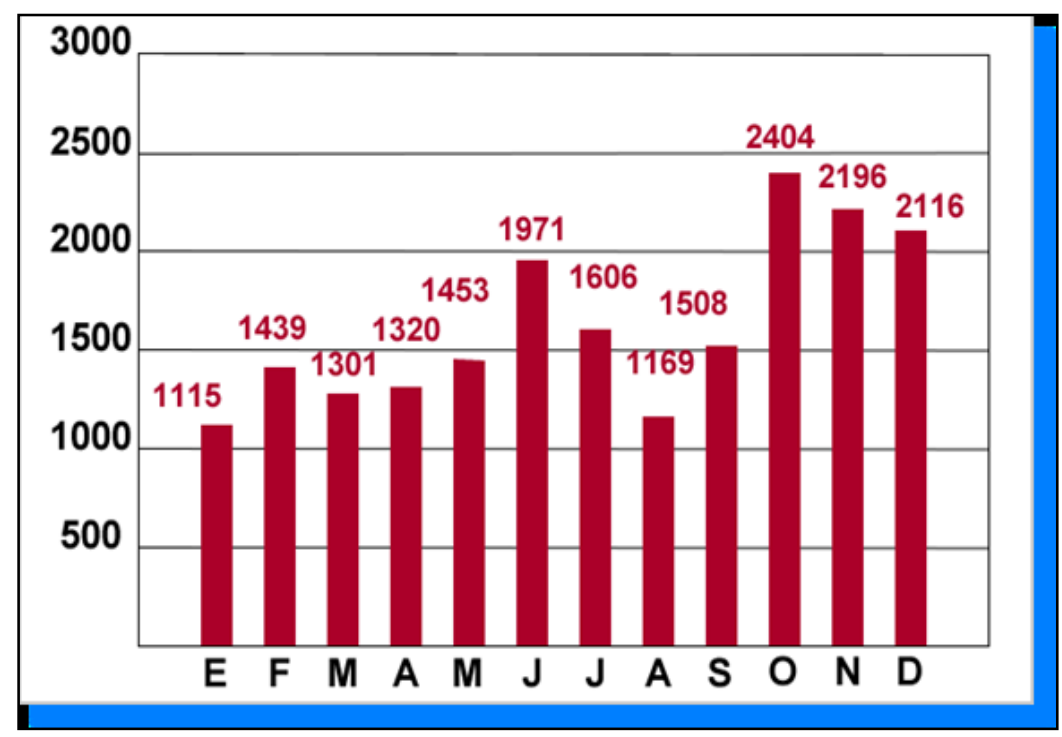

Fig. 5. Depósitos en Digital.CSIC durante el año 2012

Entre la Oficina Técnica y la Red de Bibliotecas trasvasamos unos 4.300 registros a través de la pasarela, siendo las tipologías documentales más trasvasadas los artículos, capítulos de libro, informes técnicos y las comunicaciones de congresos. 
MEI, II, Vol. 4, nº 6, pág. 49

Tabla 1. Tipologías documentales contenidas en Digital CSIC(2012)

\begin{tabular}{|l|c|}
\hline \multicolumn{1}{|c|}{ Tipologias } & N $^{0}$ registros \\
\hline Artículo & 15,862 \\
\hline Capítulo de libro & 692 \\
\hline Informe Técnico & 623 \\
\hline Comunicación de congreso & 600 \\
\hline Patente & 328 \\
\hline Póster & 212 \\
\hline Tesis & 179 \\
\hline Trabajo de divulgación & 163 \\
\hline Pre-print & 141 \\
\hline Libro & 125 \\
\hline Presentación & 109 \\
\hline Reseña bibliográfica & 84 \\
\hline Partitura & 81 \\
\hline Documento de trabajo & 69 \\
\hline material didáctico & 55 \\
\hline Tesina & 17 \\
\hline Dataset & 15 \\
\hline Video & 14 \\
\hline Imagen & 10 \\
\hline Software & 5 \\
\hline Proyecto fin de carrera & 4 \\
\hline
\end{tabular}

Este tipo de carga convive con el método tradicional, es decir, para depositar en Digital.CSIC es necesario rellenar seis pantallas de metadatos a través del software DSpace, con lo que se pierde más tiempo y hay más posibilidad de error a la hora de rellenar los campos.

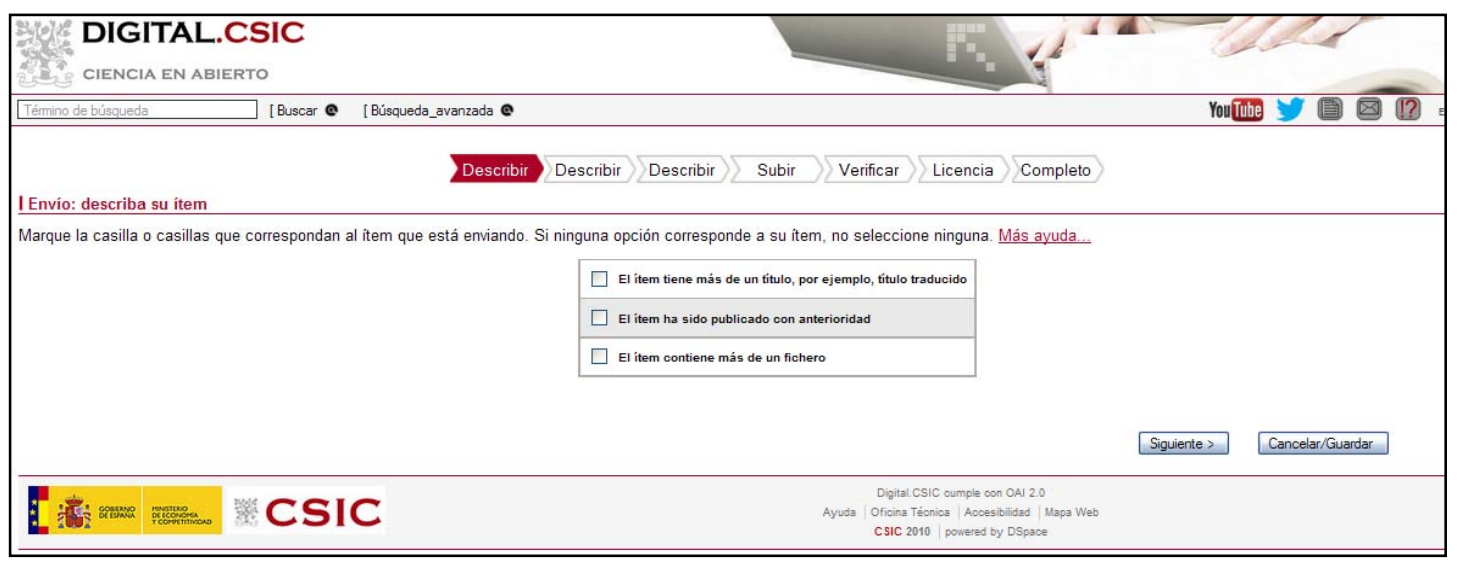

Fig. 6. Pantalla para el depósito en Digital.CSIC 
En el mes de junio se añadió información bibliométrica a nivel de ítem en Digital.CSIC con el objetivo de enriquecer los datos sobre estadísticas de uso que proporciona el repositorio para ofrecer de una manera más completa el impacto de los trabajos depositados desde distintos indicadores:

- A nivel de artículo se añadió el API de Scopus para poder recuperar y visualizar el número de citas científicas recogidas por Scopus. Por ejemplo, si se pincha en "Cited 6 times in Scopus" te direcciona a la plataforma de Scopus con el listado de esos 6 trabajos, en el caso de que no se esté suscrito sólo se puede visualizar ese listado.

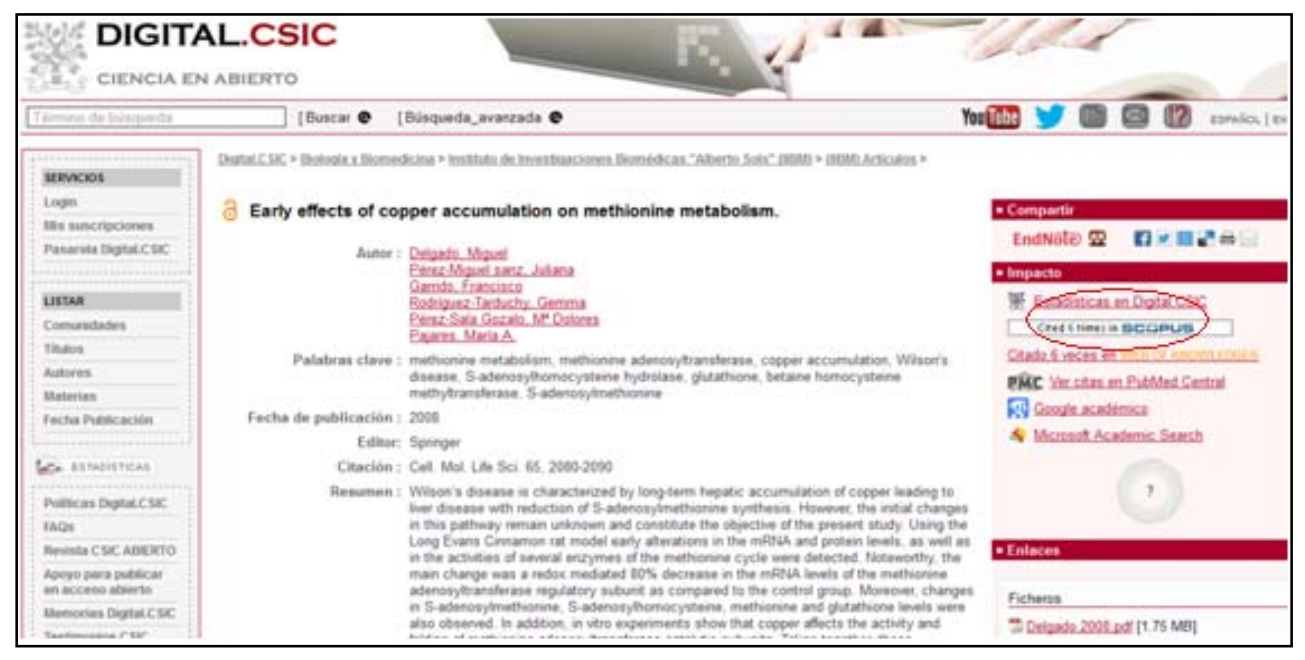

Fig. 7. Estadísticas en DIGITAL.CSIC

- Se incorporó el API de Mendeley que permite exportar referencias a aquellos investigadores que tengan cuenta en esta aplicación web, además pueden añadir en su biblioteca de Mendeley los datos relativos a sus trabajos tal y como vayan depositándose en el repositorio.

- La instalación del software Flowplayer permitió visualizar dentro de los registros bibliográficos los vídeos alojados en Digital.CSIC.

- La incorporación al Discovery tool institucional en la que todos los contenidos de Digital.CSIC se indexaron en Summon, software perteneciente a la empresa Serials Solutions, utilizado en la Biblioteca Virtual de la Red de Bibliotecas del CSIC. 
MEI, II, Vol. 4, nº 6, pág. 51

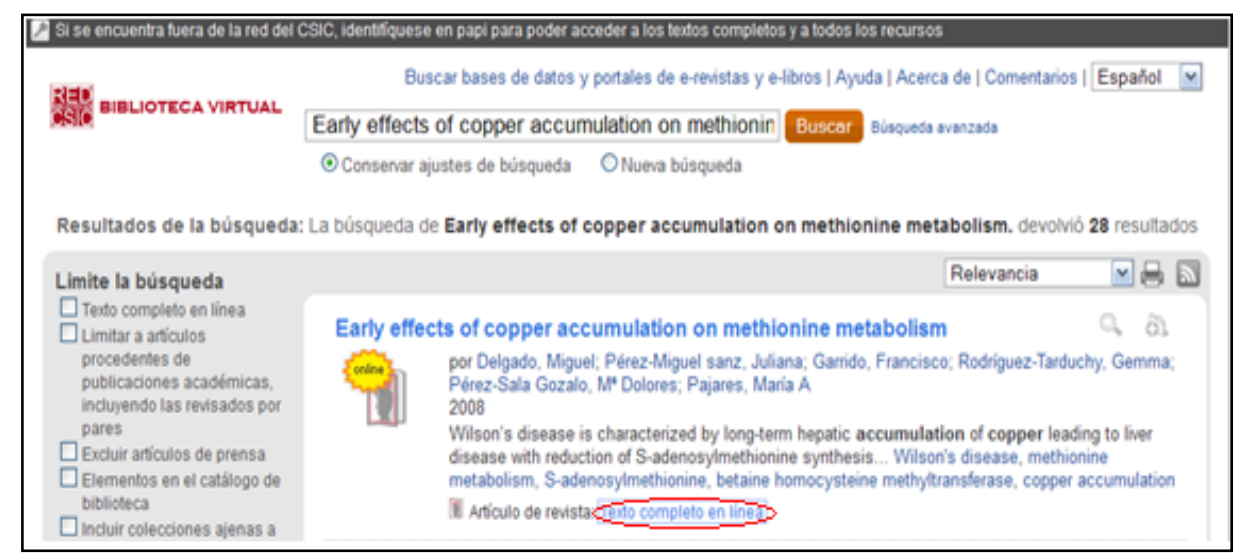

Fig. 8. Biblioteca Virtual del CSIC

- A nivel de ítem, se puede reunir las citas de un trabajo recogido por Google Scholar clicando en su logo. También se ha enlazado a Microsoft Academic Search, el buscador gratuito de información científica de Microsoft. Ambos enlaces funcionan con las siguientes tipologías documentales (dc.type): artículo, libro, comunicación de congreso, patente y preprint.

En el 2013 se ha mejorado el rendimiento de las estadísticas para que las consultas sean más rápidas, además también se ha incluido en el módulo de estadísticas un apartado nuevo que mide la producción científica depositada por totales, por centros/ institutos, por tipología documental, por año de depósito y por áreas científicas, con el objetivo de analizar la evolución del repositorio, y establecer donde se concentra el mayor volumen de trabajos accesibles gratuitamente desde el repositorio. Estas estadísticas recogen los registros cuyo metadato dc.rights esté bien formulado, es decir, es necesario seleccionar cualquiera de estas tres opciones openAccess, closedAccess o embargoAccess, porque de lo contrario habría errores en las estadísticas de Digital.CSIC.

A nivel de registro se ha modificado la visualización de las fichas de cada registro y se ha separado la descripción en metadatos de las funcionalidades en torno a los trabajos en sí, de esta manera se hace más clara la presentación de toda la información. Además se ha añadido a los registros el símbolo internacional del acceso abierto con sus posibles variantes (acceso abierto, acceso restringido, embargo).

Se han realizado mejoras en las funcionalidades en torno a los registros organizados en cuatro bloques: Compartir, Impacto, Enlaces y Artículos relacionados. En el apartado de Impacto se han añadido nuevas APIs: la de la Web of Knowledge para recoger las citas, la API de Altmetrics para recoger el tráfico que ha generado el trabajo en las redes sociales (Facebook, Mendeley, Twitter, F1000, etc.) y, en los casos de producción relativa a la biomedicina, la API que recoge citas de PubMedCentral. Estas APIs, incluida la de Scopus, funcionan usando el DOI como identificador para recuperar información en la web. Como resultado el repositorio Digital.CSIC aparte de ser una herramienta de difusión gratuita también sirve para analizar el im- 
pacto de la producción depositada a través de las estadísticas, citas y otros indicadores.

En cuanto a nuevas líneas de funcionalidades para el futuro, aparte de estas mejoras realizadas en Digital.CSIC, se está trabajando desde la Oficina Técnica para establecer una política de acceso abierto institucional, realizar la migración a DSpace 3.0 así como a la versión 2.0 de ConCiencia, consolidar el Plan 100\% Digital para apoyar los centros/institutos que no tienen personal bibliotecario y así continuar con la carga de la producción científica en el repositorio, participar en varios proyectos internacionales de acceso abierto, introducir más sinergias con ConCiencia a través de la reutilización de datos CVN o el uso de Digital.CSIC como fuente de datos y, por último, elaborar un programa de soporte para Horizon2020 (2014-2020).

\section{5.-Conclusión.}

Desde que el repositorio Digital.CSIC comenzó su andadura se ha observado un incremento importante en el número de registros depositados, sobre todo, en este último año con la implementación de la pasarela ConCiencia, cuya tipología documental predominante sigue siendo el artículo. Aunque existen investigadores que están a favor del acceso abierto, también hay quienes están totalmente en contra, ya sea, por cuestiones estéticas (pre-print), por desconocimiento de lo que es el acceso abierto o por haber cedido sus derechos de explotación, por lo que es necesario seguir informando de los beneficios que pueden obtener los investigadores al archivar sus trabajos en un repositorio de acceso abierto y, concienciarlos de que los mandatos hay que cumplirlos.

Por último, las nuevas funciones incorporadas al repositorio son fundamentales para la evaluación, gestión y difusión de la producción institucional.

\section{6.-Referencias bibliográficas}

BERNAL, I. y OFICINA TÉCNICA DE DIGITAL.CSIC (2012). Digital.CSIC. Medida, visibilidad $e$ impacto de la producción científica [en línea]. Madrid: CSIC. Disponible en:ç <http://digital.csic.es/ bitstream/10261/49948/1/ Curso2012_DigitalCSIC_Impacto.pdf> [Consulta 26 Junio 2013].

BERNAL, I. y OFICINA TÉCNICA DE DIGITAL.CSIC (2012). Digital.CSIC. Acceso abierto a la producción científica del CEQMA (ICMA, ISQCH) y servicios para sus investigadores. Políticas, leyes y mandatos de acceso abierto y obligaciones contractuales de la comunidad científica del CSIC [en línea]. Madrid: CSIC. Disponible en:

$<$ http://digital.csic.es/bitstream/10261/61131/1/ CEQ MA_DIGITALCSIC.pdf> [Consulta 28 Junio 2013].

BERNAL, I. y OFICINA TÉCNICA DE DIGITAL.CSIC (2013). "Los resultados de la ciencia, accesibles para todos los públicos". Sociedad Española de Bioquímica y Biología Molecular. Disponible en: $<$ http://www.sebbm.es/ES/divulgacion-ciencia-para-todos_10/los-resultados-de-la-ciencia-- accesibles-para-todos_889> [Consulta 1 Julio 2013].

BERNAL, I. y OFICINA TÉCNICA DE DIGITAL.CSIC (2013). Digital.CSIC. Impacto de la cien cia en acceso abierto [en línea]. Madrid: CSIC. Disponible en:

<http://digital.csic.es/ bitstre am/10261/73245/1/DC_impacto_oa_ciencia_bernal_2013.pdf> 
MEI, II, Vol. 4, nº 6, pág. 53

[Consulta 28 Junio 2013].

COMISIÓN EUROPEA (2012). Open Access in FP7 [en línea]. Disponible en:

$<$ http://ec.europa.eu/research/press/2008/pdf/annex_1_new_clauses.pdf> [Consulta 28 Junio 2013]

SIC (2013). Publicación en acceso abierto [en línea]

http://bibliotecas.csic.es/publicacion-en- acceso-abierto [Consulta 30 Junio 2013].

EUROPEAN RESEARCH COUNCIL (2012). Open Access Guidelines for researchers funded by the ERC [en línea]. Disponible en:

<http://erc.europa.eu/sites/default/files/document/file/

open_access_policy_researchers_funded_ERC.pdf $>$ [Consulta 28 Junio 2013].

OFICINA TÉCNICA DE DIGITAL.CSIC (2011). Digital.CSIC. Aumenta el impacto de tu investiga ción [en línea]. Madrid: CSIC. Disponible en:

<http://digital.csic.es/bitstream/10261/41579/2/ dc_impacto_espanol.pdf> [Consulta 29 Junio 2013].

OFICINA TÉCNICA DE DIGITAL.CSIC (2013). Digital.CSIC Ciencia en Abierto. Memoria 2012 [en línea]: Madrid: CSIC. Disponible en:

<http://digital.csic.es/bitstream/10261/78724/3/Me moria_Digital_CSIC_2012.pdf> [Consulta 28 Junio 2013].

OFICINA TÉCNICA DE DIGITAL.CSIC (2013). Listado de proyectos CSIC afectados por políticas de acceso abierto del 7PM y ERC [en línea]. Madrid: CSIC. Disponible en:

$<$ https://digital.csic.es/handle/10261/73668> [Consulta 28 Junio 2013].

ROMÁN-MOLINA, J. y OFICINA TÉCNICA DE DIGITAL.CSIC (2013). Digital.CSIC. Desarrollo de contenidos [en línea]. Madrid: CSIC. Disponible en:

<http://digital.csic.es/ bitstre am/10261/73238/1/DC_desarrollo_contenidos_roman_2013.pdf> [Consulta 1 Julio 2013]. 